\title{
Quality of life in patients with bone tumour
}

\author{
Olga Shchelkova and Ekaterina Usmanova \\ Saint Petersburg State University, Russian Federation
}

\begin{abstract}
Introduction - in the last decades, scientific research of health related quality of life (QoL) is developing fast worldwide. QoL concept pays attention to emotional experience of disease in patients, particularly to personal sense of possibility to satisfy actual needs and possibility of full social functioning in spite of disease specific limitations. The aim of the study was to reveal the basic aspects and personality factors of QoL in patients with bone tumour. Results: the study revealed that patients with bone metastases assess their health significantly worse than other patients. Besides, patients with osteosarcoma evaluate their general health higher than patients with giant cell tumour. Social functioning in patients with chondrosarcoma is higher than in patients with bone metastases and patients with giant cell tumour. Patients with chondrosarcoma have higher physical functioning and less restricted in daily activities than patients with bone metastases. Patients with bone metastases have the most intensive pain among all patients. Moreover, the study revealed personality significant influence on QoL related to bone tumour. Conclusion - patients with bone metastasis have lower QoL compared to patients with primary bone tumours. Patients with giant cell tumour have the worth quality of life among patients with primary bone tumours. Furthermore the results revealed differences in QoL parameters associated with personality characteristics in patients with bone tumour.
\end{abstract}

Key words: quality of life, bone tumour, oncologic patients, side effects of the therapy.

\section{Introduction}

The issue of quality of life in oncologic patients is widely discussed problem in present-day science. General health of a patient may be complicated with basic disease manifestations and side effects of the therapy [1]. In the last decades scientific research of health related quality of life has developed fast worldwide [1-5]. Especially researchers focus on the quality of life in oncologic patients seeing real burden of the disease, vital threat often following the illness, and frequency of psychological problems in oncologic patients [2].

QoL concept pays attention to emotional experience of the disease in patients, particularly to personal sense of possibility to meet the current needs and possibility of full social functioning in spite of the disease specific limitations. Studies of QoL in patients with bone tumour are aimed at research QoL in children and adolescents with bone sarcoma. There are also studies aimed at comparative analysis of QoL in patients with bone tumour who underwent limb sparing surgery and amputation. Issue of QoL in adults with bone tumour focused on psychological factors of QoL is not discussed.

Without doubt, the malignant bone tumour dramatically changes patients' quality of life. Development of bone tumour is accompanied by pain and sometimes by bone fracture in the place of pathological process localization, besides it impacts on the mobility of the affected 
limb, which leads to sharp decrease of possible physical loads of patient, even long periods of nothing but confinement to bed. Bone sarcoma and giant cell tumour treatment in most cases requires surgical resection of the affected part of bone and substitution of the defect with endoprosthesis.

The aim of the study was to reveal the basic aspects and personality factors of QoL in patients with bone tumour.

Patients - Study participants from 2012 to $2015(N=120)$ were in-patients with primary and metastatic bone tumours of N.N. Blokhin Russian Cancer Research Centre.

According the research goals general group was split into four groups depending on the diagnosis. The first one (A) included 41 patients diagnosed with osteosarcoma, at average age $27 \pm 2$. There were $25(61 \%)$ males and $16(31 \%)$ females in this group. The second group (B) included 31 patients with giant cell tumour with average age of $36 \pm 3$. There were $13(42 \%)$ males and 18 (58\%) females. The third group (C) included 30 patients diagnosed with chondrosarcoma at the age of $45 \pm 3$. Males prevailed in this group; they were $24(80 \%)$ patients. Females were 6 (20\%) patients. The fourth group (D) included 18 patients with bone metastases (primary diagnosis is adenocarcinoma). There were $14(78 \%)$ males and $4(22 \%)$ females. The average age is $55 \pm 2$. All patients had surgical or complex treatment.

\section{Methods}

The methods were:

1. "SF-36 Health Status Survey" was used for determining quality of life in patients with bone tumour. 36 items of the questionnaire form 8 scales: General Health (GH), Physical Functioning (PF), Role-Physical (RP), Role-Emotional (RE), Social Functioning (SF), Bodily Pain (BP), Vitality (VT), Mental Health (MH). Scores range from 0 till 100. 100 scores present the best health. The scales form two parameters "physical health" (first four scales) and "mental health" (last four scales) [6, 7].

2. "Quality of Life Questionnaire Core-30" was designed by Study Group on Quality of Life of European Organization for Research and Treatment Cancer. The use of QLQC30 shows that it is highly sensitive tools for evaluating of quality of life in patients with oncologic disease apart from its type. The modern version of EORTC QLQC30 includes 30 questions and consists of 5 functional scales (Physical functioning, Role functioning, Emotional functioning, Cognitive functioning, Social functioning), 3 symptom scales (Fatigue, Nausea and vomiting, Pain), Global health status and 6 symptom items (Dyspnoea, Insomnia, Appetite loss, Constipation, Diarrhoea, Financial difficulties) $[8,9]$.

3. Module BM22, which was used for studying of specific symptoms show quality of life in patients with malignant bone tumour. The module consists of 20 questions and has 2 Symptom scales (Painful Sites and Pain Characteristics) and 2 Functional scales (Functional Interference and Psychosocial Aspects) [8, 9].

4. Personal questionnaire "Big V" aims at revealing distinguished five global personal factors bound up with the levels of extraversion, self-consciousness, cooperation, emotional stability and personal resources $[10,11]$.

5. Method "Ways of Coping" aims at revealing coping strategies in stressful and difficult situations person uses. The strategies are confrontation, distancing, self-control, search for social support, acceptance of responsibility, escape, plan to solve the problem, positive reconsideration [12-14].

6. Method "Purpose in life" is determined by the authors as person experience of ontological life significance [15-17]. 


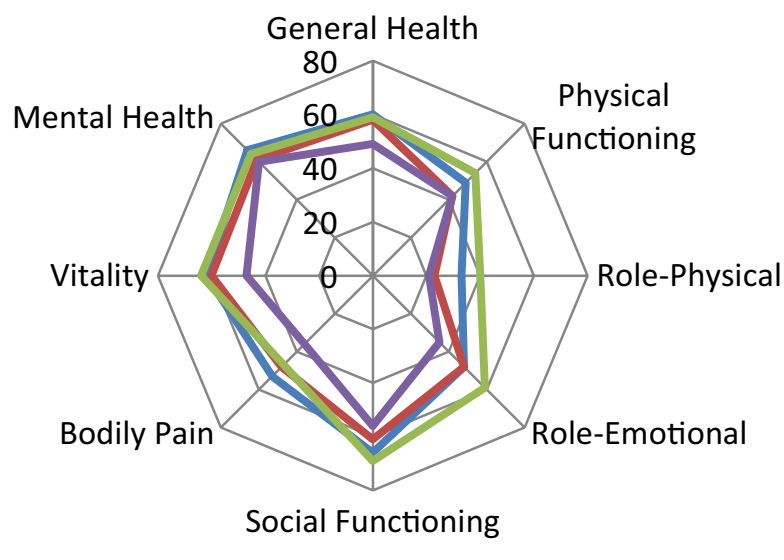

Osteosarcoma Giant cell tumor

Fig. 1. Health related quality of life indices in patients with bone tumor (SF-36).

Statistical methods - statistical significance of distinctions between groups was calculated with t-criterion Student test. Multifactoral dispersing analysis of data revealing personality impact on quality of life in four clinical groups of patients was also made.

\section{Results and discussion}

According the results of SF-36 patients with bone metastases evaluate their general health and treatment prospects significantly lower than patients with osteosarcoma.

As Fig. 1 shows, patients with chondrosarcoma have lower influence of their physical state on daily activities, than patients with giant cell tumour and bone metastases. In addition, patients with bone metastases have more restricted activity caused by pain intensity and have lower vitality than other patients.

These data are consistent and complimentary with the results of studying health related quality of life researched using SF-36. QLQ C-30 questionnaire was designed as method for quality of life assessment especially in patients with malignant tumour.

Using QLQ C-30 differences in the patient assessment of general health were revealed. As Figs. 2 and 3 show, patients with bone metastases assess their health significantly worse than other patients. Apart from that, patients with osteosarcoma evaluate their general health higher than patients having giant cell tumour.

Patients with chondrosarcoma have higher physical functioning and are less restricted in daily activities than patients with bone metastases, and these data are also consistent with the results of studying health related quality of life researched using SF-36. Social functioning in patients with chondrosarcoma is higher than in patients with bone metastases. Besides according QLQ C-30 data patients with chondrosarcoma have higher social functioning than patients with giant cell tumour.

Patients with bone metastases have the most intensity pain among all patients. According BM 22 data, quality of life in patients with bone metastases is lower than quality of life in patients with primary bone tumours on the scale "Pain Sites": patients with bone metastases characterize their pain as more widespread than other patients. Besides patients with bone metastases have more intensity pain than patients with osteosarcoma. 


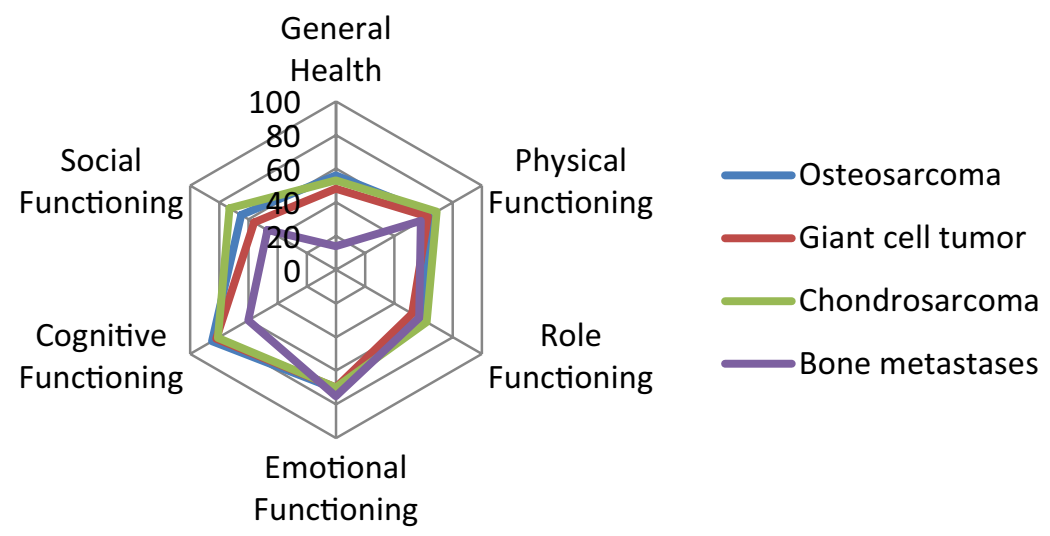

Fig. 2. Health related quality of life indices in patients with bone tumour (QLQ C-30). Functional scales.

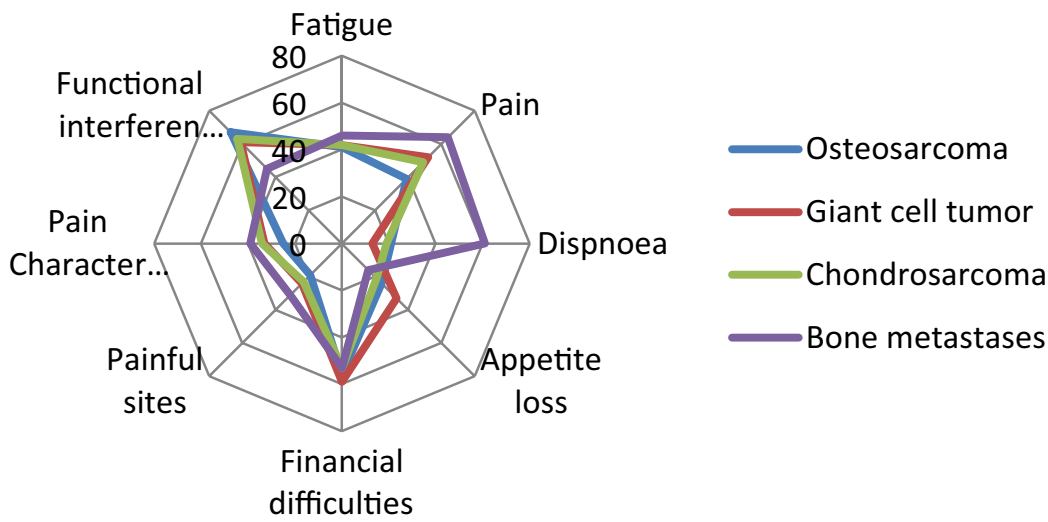

Fig. 3. Health related quality of life indices in patients with bone tumour (QLQ C-30 and BM 22). Symptom scales.

Moreover, the study revealed significant influence of the personality on QoL related to bone tumour.

Factorial analysis of indices of questionnaires "Big V", "Ways of Coping Behaviour" and "Meaning of life orientations" was conducted. Consequently, these factors were used for further analysis. In the next stage in the result of cluster analysis, personality profiles in patients were revealed. The profiles include psychological characteristics the factors have.

As you can see in Fig. 4 four groups of patients with bone tumour were revealed in the result of cluster analysis. The first group includes patients with high personal resources, disposition to cooperation and positive revaluation. This cluster was called "Person aimed at adjustment".

The second group includes patients with low personal resources, disinclination to problem solving behaviour, low consciousness and social dependence. The cluster was called "Addicted person". 


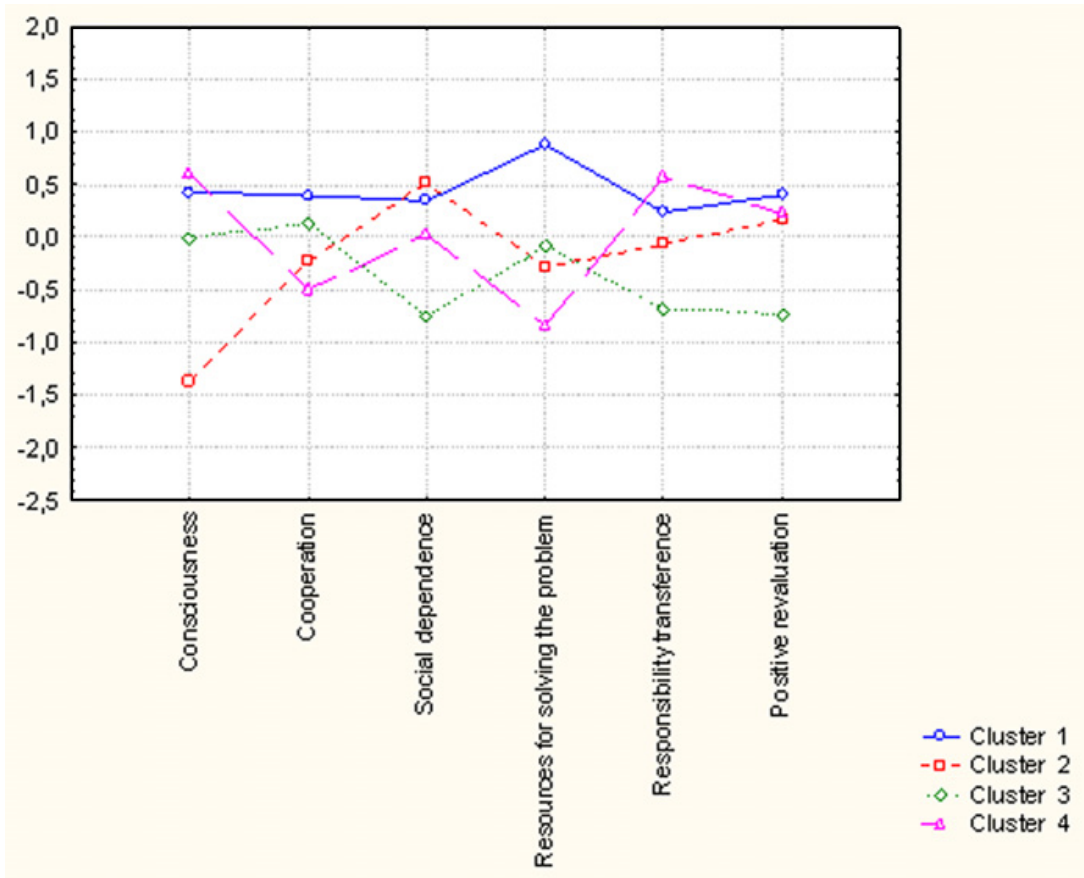

Fig. 4. Clusters of personality in patients with bone tumour.

The third group consists of patients with bone tumour not inclined to responsibility transference, not socially addicted, meanwhile not inclined to positive revaluation. The third cluster was called "Responsibility and alienation".

The fourth group includes patients with high level of consciousness, inclined to responsibility transference and not inclined to problem solving behaviour. This cluster was called "Egocentrism and alienation".

In the next stage, dispersion analysis was made with the purpose of study influence the personality profiles on QoL.

As Fig. 5 shows, patients of the first cluster "Person aimed at adjustment" have the highest quality of life compared with other patients. Patients of the second cluster have the lowest quality of life among patients with bone tumour.

Cluster "Person aimed at adjustment" differs significantly from cluster "Addicted person" on the factor "Consciousness". Consequently, the factor of consciousness has the most important value in the issue of influence psychological characteristics on quality of life in patients with bone tumour.

Besides, there are differences between these clusters on factors "Cooperation", "Positive revaluation", "Resources for solving problems" and "Social Dependence".

\section{Conclusions}

Quality of life and general health status in patients with primary bone tumour is significantly better than quality of life and general health status in patients with bone metastases. There are differences in QoL parameters between patients with primary bone tumour: patients with giant cell tumour have the worst QoL among patients with primary bone tumour and the same general health status. 


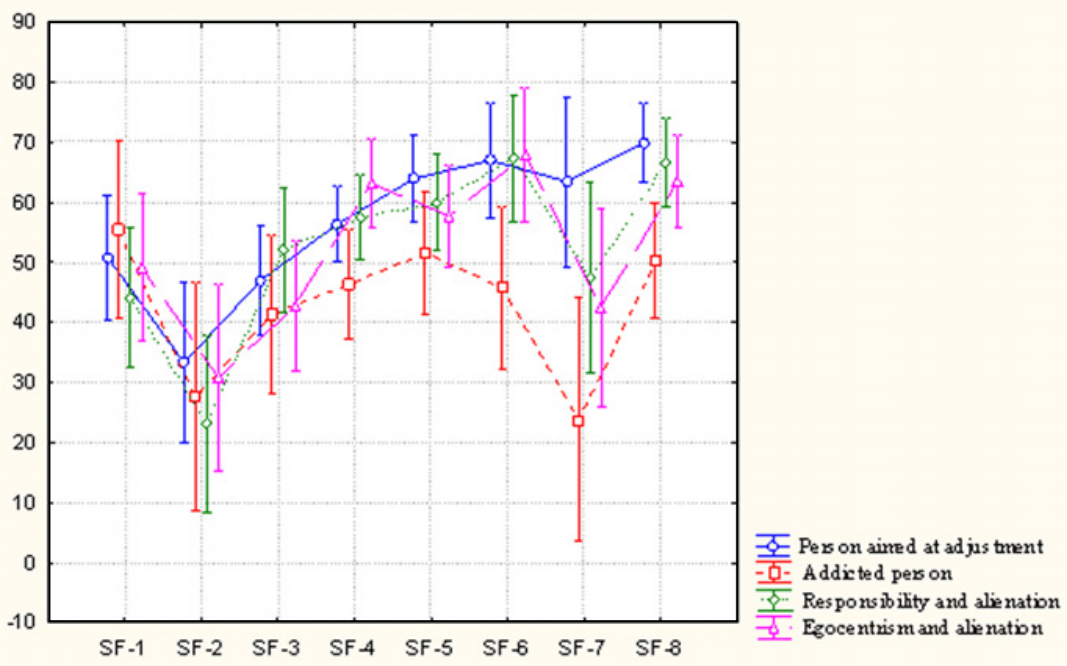

Fig. 5. Influence of personality profiles on quality of life in patients with bone tumour.

In addition, psychological factors, which influence quality of life, are revealed. First of all, patients with high level of consciousness and the significance of life have the highest quality of life among patients with bone tumour.

Such characteristics in structure of personality as high degree of self-consciousness, personal resources, cooperation and disposition to positive revaluation in difficult situation correspond to higher QoL. On the contrary, low personal resources and slight problem solving behaviour, low degree of self-consciousness and high social dependence correspond to decrease in QoL in patients with bone tumour.

RFFI grant No. 17-36-00011 “a1”.

\section{References}

[1] N.K. Aaronson, S. Ahmedzai, B. Bergman, J. Natl. Cancer Inst. 85, 365-375 (1993)

[2] G.H. Christ, J.M. Lane, R. Marcove, J. Psychosocial Oncol. 13, 1-21 (1995)

[3] J.C. Crumbaugh, L.T. Maholick, J. Clin. Psychology 20, 200-207 (1964)

[4] J.C. Crumbaugh, L.T. Maholick, Manual of instructions for the Purpose in Life test (Viktor Frankl Institute of Logotherapy, TX, Abilene, 1969)

[5] C. Eiser, A.E. Darlington, S.B. Stride, R.J. Grimer, Quality of life implications as a consequence of surgery: limb salvage, primary and secondary amputation. Sarcoma $\mathbf{5}$, 189-195 (2001)

[6] C. Eiser, Europe J. Cancer 45, 1744-1777 (2009)

[7] P. Fayers, N. Aarson, K. Bjordal, M. Sullivan, QLQ C-30 Scoring Manual (EORTC Study Group on Quality of Life, Brussels, 1995)

[8] L.R. Goldberg, J. Personality Soc. Psychol. 59, 1216-1229 (1992)

[9] L.N. Kasimova, T.V. Zchiryaeva, Mental Disorders Medicine 1, 16-19 (2009)

[10] R.S. Lazarus, Stress and the coping: An anthology, 2 ed. (Columbia University Press, New York, 1985), p. 437 
[11] R.S. Lazarus, S. Folkman, Stress, appraisal and coping (Springer, New York, 1984), p. 456

[12] D.A. Leontyev, The test of meaningful orientations (Smysl, Moscow, 2000), p. 18

[13] R. Oren, A. Zagury, O. Katzir, Y. Kollender, I. Meller, Musculoskeletal Cancer Surgery. Treatment of Sarcomas and Allied Disease, 581-586 (2001)

[14] L.I. Wasserman, E.A. Trifonova, A.YU. Shelkova, Psychological diagnostics and intervention in physical clinic. S-Pet: Speech (2011)

[15] L.I. Wasserman, V.A. Ababkov, E.A. Trifonova, Coping: theory and psychodiagnostics, SPb. Rech, p. 192 (2010)

[16] J.E. Ware, K.K. Snow, M. Kosinski, B. Gandek, SF-36 Health Survey Manual and Interpretation Guide (New England Medical Center, The Health Institute, MA, Boston, 1993)

[17] D.P. Yanichev, Cognitive aspects of personal characteristics in patients with neurotic symptoms (2006) 